\title{
$\tau$ decay into a pseudoscalar and an axial-vector meson
}

\author{
L. R. Dai, ${ }^{1,2, *}$ L. Roca, ${ }^{3, \dagger}$ and E. Oset ${ }^{2, *}$ \\ ${ }^{1}$ Department of Physics, Liaoning Normal University, Dalian 116029, China \\ ${ }^{2}$ Departamento de Física Teórica and IFIC, Centro Mixto Universidad de Valencia-CSIC, \\ Institutos de Investigacíon de Paterna, Aptdo. 22085, 46071 Valencia, Spain \\ ${ }^{3}$ Departamento de Física, Universidad de Murcia, E-30100 Murcia, Spain
}

(Received 11 December 2018; published 6 May 2019)

\begin{abstract}
We study theoretically the decay $\tau^{-} \rightarrow \nu_{\tau} P^{-} A$, with $P^{-}$a $\pi^{-}$or $K^{-}$and $A$ an axial-vector resonance $b_{1}(1235), h_{1}(1170), h_{1}(1380), a_{1}(1260), f_{1}(1285)$ or any of the two poles of the $K_{1}(1270)$. The process proceeds through a triangle mechanism where a vector meson pair is first produced from the weak current and then one of the vectors produces two pseudoscalars, one of which reinteracts with the other vector to produce the axial resonance. For the initial weak hadronic production we use a recent formalism to account for the hadronization after the initial quark-antiquark pair produced from the weak current, which explicitly filters $G$-parity states and obtain easy analytic formulas after working out the angular momentum algebra. The model also takes advantage of the chiral unitary theories to evaluate the vector-pseudoscalar (VP) amplitudes, where the axial-vector resonances were obtained as dynamically generated from the vectorpseudoscalar interaction. We make predictions for invariant mass distribution and branching ratios for the channels considered.
\end{abstract}

DOI: 10.1103/PhysRevD.99.096003

\section{INTRODUCTION}

The fact that the $\tau$ is the only lepton heavy enough to decay into hadrons makes the hadronic $\tau$ lepton decays a priceless test of the strong interaction at low energy in the light flavor sector [1-4]. The intermediate and final state decay hadrons are usually produced with lower background than in other low energy processes. Even though there are more than 100 hadronic $\tau$ decays experimentally reported by the PDG [5] (which account for about $65 \%$ of the $\tau$ decay width), it is also clear that not all the possible ones have been observed or whether there is no room for decays beyond the standard model. While inclusive reactions are well suited for accurate extraction of standard model parameters such as the strong coupling constant $[3,6,7]$, the exclusive ones are much more involved and difficult to predict within QCD, and here is where effective theories for hadronic low energy interactions gain prominence. Special theoretical attention has been devoted to decay channels with two and three pseudoscalar mesons in the final state (see [8] for a brief review). Channels with more

\footnotetext{
*dailr@lnnu.edu.cn

†luisroca@um.es

*oset@ific.uv.es
}

Published by the American Physical Society under the terms of the Creative Commons Attribution 4.0 International license. Further distribution of this work must maintain attribution to the author(s) and the published article's title, journal citation, and DOI. Funded by SCOAP. pseudoscalars or other mesons like vector or axial-vector ones are less studied $[1,9]$. Particularly, very poorly understood are the channels with one pseudoscalar plus one axial-vector meson in the final state, which are the aim of the study in the present work. Experimentally only the $f_{1}(1285) \pi$ channel has been measured [5]. It is at this point where effective theories of strong interactions at low energies can stand up. Particularly, the unitary extensions of chiral perturbation theory $(\mathrm{U} \chi \mathrm{PT})$ provide a dynamical and powerful explanation of the generation of the low-lying axial-vector resonances [10-12]. With the only input of the lowest order chiral perturbation theory Lagrangians and the implementation of unitarity in coupled channels, most of the lowest mass axial vector resonances $\left[b_{1}(1235)\right.$, $h_{1}(1170), h_{1}(1380), a_{1}(1260), f_{1}(1285)$ and two poles for the $K_{1}(1270)$ ] were dynamically obtained [10-12] as poles in the pseudoscalar-vector (PV) scattering amplitudes, without the need to include them as explicit degrees of freedom. With only one free parameter (for regularization of PV loops), this model predicts not only masses and widths of these axial vector resonances but also the full PV scattering amplitudes from where e.g., the coupling of the different resonances to the different PV channels can be obtained. Within this model, the $\tau$ decay into one pseudoscalar plus one axial-vector resonance requires the production of one pseudoscalar and one PV pair in the hadronization process, since these axial-vector resonances are dynamically generated from the PV interaction. This can be dominantly accomplished via a triangular 


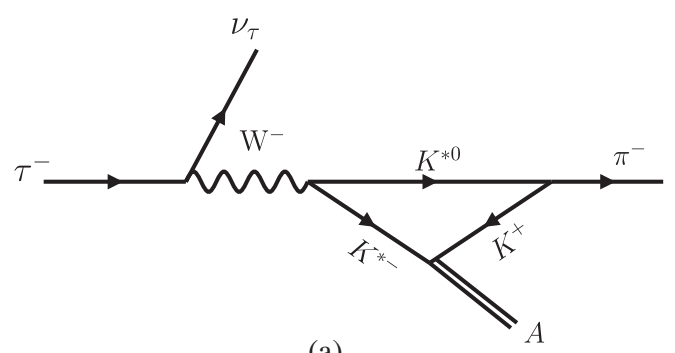

(a)

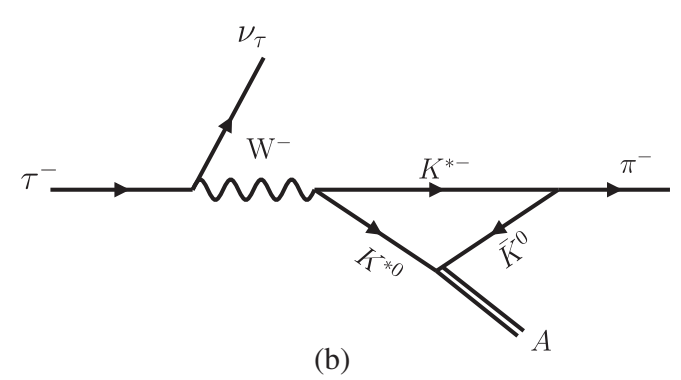

(b)

FIG. 1. Diagrams for the decay of $\tau^{-} \rightarrow \nu_{\tau} \pi^{-} A$, with $A$ axial vectors.

mechanism of the kind shown in Fig. 1. Actually, for the $f_{1}(1285) \pi$ case, it was shown in [13] that it was the dominant contribution. Indeed, for some particular kinematic conditions, the triangle diagram benefits from a large enhancement since it develops a singularity [14], which according to the Coleman Norton theorem [15] is related to the classical process in which a particle decays into two particles $\mathrm{A}$ and $\mathrm{B}$, then $\mathrm{A}$ decays into two other particles, and one of them merges with particle B to produce a third particle. A new reformulation of these findings can be seen in [16,17]. A similar mechanism has been also recently used for the decay $\tau^{-} \rightarrow$ $\nu_{\tau} \pi^{-} f_{0}(980)\left(a_{0}(980)\right)$ [18]. Other theoretical approaches regarding the $\tau$ decay into an axial and a pseudoscalar can be found in [22-24] where the axial is produced as an intermediate resonance produced in the weak vertex.

On the other hand, for the hadronization process from the $W^{-}$boson to two mesons, we follow the approach of [19], where the ${ }^{3} P_{0}$ model $[20,21]$ is used to hadronize the primary quarks produced from the weak interaction, working out all the angular momentum and spin algebra. In particular, a Cabibbo favored $d \bar{u}$ pair is produced from the $W^{-}$which then hadronizes from an extra $q \bar{q}$ pair with the quantum numbers of the vacuum. The different possible final meson-meson states are related by $S U(3)$ symmetry. The strength of the formalism in [19] is that it carries on an elaborate calculation of the angular momentum and spin algebra which allows, in the end, to rely upon only one global unknown constant to get all the different channels. This unknown factor is obtained in the present work from the experimental value of the $\tau^{-} \rightarrow \nu_{\tau} K^{*} \bar{K}^{*}$ decay. Furthermore, the formalism allows for an explicit filter of $G$-parity states, of special importance in the present work.

\section{FORMALISM}

\section{A. Feynman diagrams}

We are going to study the decays $\tau^{-} \rightarrow \nu_{\tau} \pi^{-} A$, with $A$ being axial-vector resonances, including the positive $G$ parity $f_{1}(1285), b_{1}(1235)$ states, and negative $G$-parity $h_{1}(1170), h_{1}(1380)$ and $a_{1}(1260)$ states, and the $\tau^{-} \rightarrow$ $\nu_{\tau} K^{-} K_{1}(1270)$ decay. As mentioned in the Introduction, these were the low-lying axial-vector resonances dynamically generated in [11]. Actually, for the $K_{1}(1270)$ resonance, it was shown in $[11,25]$ that is has a two pole structure and then we will consider both of them. Since the $A$ resonances are dynamically generated from the PV interaction, for the $\tau^{-} \rightarrow \nu_{\tau} P^{-} A$, the way to produce the vector-pseudoscalar (VP) to generate axials and the extra pseudoscalar in the final state is via a triangular mechanism of the kind shown in Fig. 1. Other possible diagrams with a different combination of pseudoscalar and vector mesons inside the triangle loop are also possible but they need VVP or pseudoscalar-pseudoscalar-pseudoscalar (PPP) anomalous vertices which are small. Furthermore, diagrams with analogous triangles but where two pseudoscalars are produced from the $W$ boson and with a vector in the right leg of the triangle are also small. Indeed, in the case of $K \bar{K} K^{*}$ in the loop, it requires the coupling of the $W$ to $K \bar{K}$ which must be about one order of magnitude smaller than to $K^{*} \bar{K}^{*}$ as can be inferred from the ratio of phase space allowed for the $\tau^{-} \rightarrow \nu_{\tau} K \bar{K}$ and $\tau^{-} \rightarrow \nu_{\tau} K^{*} \bar{K}^{*}$ and the experimental results. For the case of the $\pi \pi \rho$ in the loop, with the $W$ boson coupling to $\pi \pi$ and a $\rho$ in the other leg of the loop, which contributes for negative $G$-parity axial production, the justification of its small size is not so clear. Therefore we will evaluate its explicit contribution explicitly later on.

The well-defined $G$-parity axial-vector states have dominant couplings to either $\rho \pi$ or $K^{*} \bar{K}$ [11]. Therefore, for the positive $G$-parity axial-vector states $\left[f_{1}(1285)\right.$ and $\left.b_{1}(1235)\right]$, the complete Feynman diagrams for the decay with the triangle mechanism are those shown in Fig. 1. Figure 1(a) shows the process $\tau^{-} \rightarrow \nu_{\tau} K^{* 0} K^{*-}$ followed by the $K^{* 0}$ decay into $\pi^{-} K^{+}$and the merging of the $K^{*-} K^{+}$ into $A$; and Fig. 1(b) shows the process $\tau^{-} \rightarrow \nu_{\tau} K^{*-} K^{0}$ followed by the $K^{*-}$ decay into $\pi^{-} \bar{K}^{0}$ and the merging of the $K^{* 0} \bar{K}^{0}$ into $A$. The momenta assignment for the decay

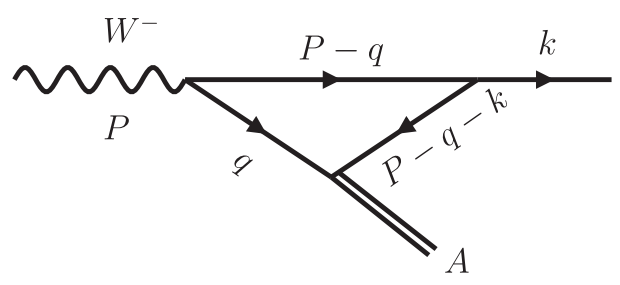

FIG. 2. The momenta assignment for the decay process. 
TABLE I. Couplings for positive $G$-parity states [11,13] (All the units are MeV).

\begin{tabular}{lcccr}
\hline \hline & \multicolumn{2}{c}{$f_{1}(1285)$} & \multicolumn{2}{c}{$b_{1}(1235)$} \\
\cline { 2 - 5 } Couplings & $g_{i}$ & $\left|g_{i}\right|$ & $g_{i}$ & $\left|g_{i}\right|$ \\
\hline$\frac{1}{\sqrt{2}}\left(\left|\bar{K}^{*} K\right\rangle_{I=0}+\left|K^{*} \bar{K}\right\rangle_{I=0}\right)$ & $7350+i 0$ & 7350 & $\ldots$ & $\ldots$ \\
$\frac{1}{\sqrt{2}}\left(\left|\bar{K}^{*} K\right\rangle_{I=1}+\left|K^{*} \bar{K}\right\rangle_{I=1}\right)$ & $\ldots$ & $\ldots$ & $6172-i 75$ & 6172 \\
\hline \hline
\end{tabular}

TABLE II. The same as Table I but for negative $G$-parity states.

\begin{tabular}{|c|c|c|c|c|c|c|}
\hline \multirow[b]{2}{*}{ couplings } & \multicolumn{2}{|l|}{$h_{1}(1170)$} & \multicolumn{2}{|c|}{$h_{1}(1380)$} & \multicolumn{2}{|c|}{$a_{1}(1260)$} \\
\hline & $g_{i}$ & $\left|g_{i}\right|$ & $g_{i}$ & $\left|g_{i}\right|$ & $g_{i}$ & $\left|g_{i}\right|$ \\
\hline$\rho \pi$ & $-3453+i 1681$ & 3840 & $648-i 959$ & 1157 & $-3795+i 2330$ & 4453 \\
\hline$\frac{1}{\sqrt{2}}\left(\left|\bar{K}^{*} K\right\rangle_{I=0}-\left|K^{*} \bar{K}\right\rangle_{I=0}\right)$ & $781-i 498$ & 926 & $6147+i 183$ & 6150 & $\cdots$ & $\cdots$ \\
\hline 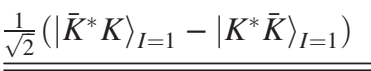 & $\ldots$ & $\ldots$ & $\cdots$ & $\ldots$ & $1872-i 1486$ & 2390 \\
\hline
\end{tabular}

process is given in Fig. 2. The needed couplings obtained in $[11,13]$ for positive $G$-parity axial-vector states to the appropriate $G$-parity VP eigenstates are given in Table I.

For the negative $G$-parity axial-vector states $\left[h_{1}(1170)\right.$, $h_{1}(1380)$ and $a_{1}(1260)$ ], we need to consider the diagrams in Fig. 3 in addition to those in Fig. 1 because $\rho \rho$ has $G=+$ and, the fact that $G(\pi)=-$ demands that the axial has negative $G$ - parity to have $G$-parity conservation. This mechanism with initial $\rho \rho$ production is thus not possible for the positive $G$-parity axials $f_{1}(1285)$ and $b_{1}(1235)$. Figure 3(a) shows the process $\tau^{-} \rightarrow \nu_{\tau} \rho^{-} \rho^{0}$ followed by the $\rho^{-}$decay into $\pi^{-} \pi^{0}$ and the merging of the $\rho^{0} \pi^{0}$ into $A$; and Fig. 3(b) shows the process $\tau^{-} \rightarrow \nu_{\tau} \rho^{0} \rho^{-}$followed by the $\rho^{0}$ decay into $\pi^{-} \pi^{+}$and the merging of the $\rho^{-} \pi^{+}$into $A$. The needed couplings [11] for negative $G$-parity states are given in Table II.

In $[11,25]$ two poles for the $K_{1}(1270)$ where obtained at complex energy positions $1195-i 123 \mathrm{MeV}$ and $1284-i 73 \mathrm{MeV}$ in unphysical Riemann sheets. The lowest mass pole, which we will call in the following $K_{1}(1)$, couples mostly to $K^{*} \pi$ and the highest one, $K_{1}(2)$, to $\rho K$. The dominant couplings are shown in Table III [25].

In Figs. 4 and 5 we show the mechanisms for $\tau^{-} \rightarrow$ $\nu_{\tau} K^{-} K_{1}(1270)$ decay for the formation of the two $K_{1}(1270)$ states. Figure 4 shows the decay $\tau^{-} \rightarrow \nu_{\tau} K^{-} K_{1}(1)$ via the process $\tau^{-} \rightarrow \nu_{\tau} K^{*-} K^{* 0}$ [Fig. 5(a)] followed by the $K^{*-}$ decay into $K^{-} \pi^{0}$ and the merging of the $K^{* 0} \pi^{0}$ into $K_{1}(1)$. Figure 5 shows the decay $\tau^{-} \rightarrow \nu_{\tau} K^{-} K_{1}(2)$ via the process $\tau^{-} \rightarrow \nu_{\tau} \rho^{-} \rho^{0}$ followed by the $\rho^{-}$decay into $K^{-} K^{0}$ and the merging of the $\rho^{0} K^{0}$ into the second pole of $K_{1}, K_{1}(2)$; Fig. 5(b) shows the process $\tau^{-} \rightarrow \nu_{\tau} \rho^{0} \rho^{-}$followed by the $\rho^{0}$ decay into $K^{-} K^{+}$and the merging of the $\rho^{-} K^{+}$into the second pole of $K_{1}, K_{1}(2)$.

\section{B. The VV weak production vertex}

Next, we address the evaluation of the $\tau \rightarrow \nu_{\tau} K^{* 0} K^{*-}$, $\nu_{\tau} K^{*-} K^{* 0}$, and $\tau \rightarrow \nu_{\tau} \rho^{-} \rho^{0}, \nu_{\tau} \rho^{0} \rho^{-}$amplitudes. The production is assumed to proceed first from the Cabibbo favored $\bar{u} d$ production from the $W^{-}$which then hadronizes producing an extra $\bar{q} q$ with the quantum numbers of the vacuum, which are implemented with the ${ }^{3} P_{0}$ model [20,26,27]. In Ref. [19] the mechanism for hadronization is done in detail. Next, we summarize and adapt the formalism to the present case. The first step corresponds to the flavor combinations in the hadronization. In Ref. [19] it is shown that $d(\bar{s} s) \bar{u}=(d \bar{s}) s \bar{u}$ gives rise to $K^{* 0} K^{*-}$, while $d(\bar{u} u) \bar{u}$ and $d(\bar{d} d) \bar{u}$ give rise to $\rho^{-} \rho^{0}$ and $\rho^{0} \rho^{-}$[see Eq. (4)] of [19]). The second step corresponds to the detailed study of the spin-angular momentum algebra to

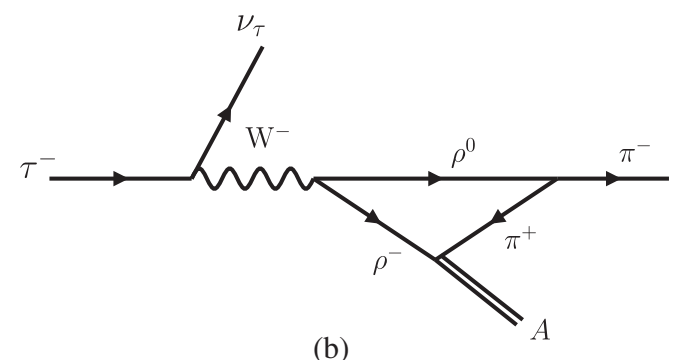

(b)

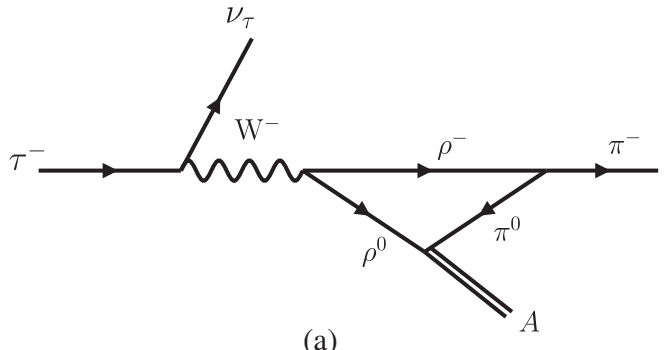

(a)

FIG. 3. The same as Fig. 1 but for $\tau^{-} \rightarrow \nu_{\tau} \rho^{-} \rho^{0}, \nu_{\tau} \rho^{0} \rho^{-}$decays. 
TABLE III. The same as Table II but for two $K_{1}(1270)$ states.

\begin{tabular}{lccccc}
\hline \hline & \multicolumn{2}{c}{$K_{1}(1)$} & & \multicolumn{2}{c}{$K_{1}(2)$} \\
\cline { 2 - 3 } \cline { 5 - 6 } couplings & $g_{i}$ & $\left|g_{i}\right|$ & & $g_{i}$ & $\left|g_{i}\right|$ \\
\hline$\rho K$ & $\ldots$ & $\ldots$ & & $5274+i 297$ & 5282 \\
$K^{*} \pi$ & $4187-i 2098$ & 4683 & & $\ldots$ & $\ldots$ \\
\hline \hline
\end{tabular}

combine the quarks for the ${ }^{3} P_{0} \bar{q} q$ state $\left(L^{\prime}=1, S^{\prime}=1\right.$, $J^{\prime}=0$ ) with a $\bar{d}$ quark in $L=1$ to have finally $s$-wave production of the two mesons. In [18] a similar calculation has been done to discuss the triangle singularity in $\tau^{-} \rightarrow$ $\nu_{\tau} \pi^{-} f_{0}(980)$ [ $\left.a_{0}(980)\right]$ decays, but with pseudoscalarvector production from the $W$ rather than two vectors, as we have here.

The elementary quark $d \bar{u}$ production in the $\tau$ decay is given by

$$
H=\mathcal{C} L^{\mu} Q_{\mu}
$$

where $\mathcal{C}$ contains the couplings of the weak interaction to be determined later on. The leptonic current is given by

$$
L^{\mu}=\left\langle\bar{u}_{\nu}\left|\gamma^{\mu}-\gamma^{\mu} \gamma_{5}\right| u_{\tau}\right\rangle
$$

and the quark current by

$$
Q^{\mu}=\left\langle\bar{u}_{d}\left|\gamma^{\mu}-\gamma^{\mu} \gamma_{5}\right| v_{\bar{u}}\right\rangle
$$

In the evaluation of the decay widths to three final particles, we find convenient to evaluate the matrix elements in the

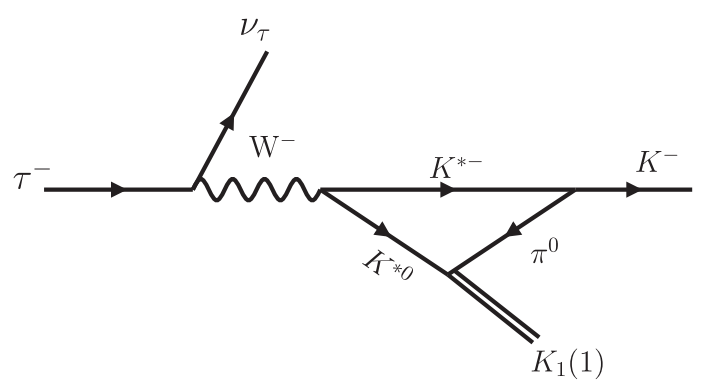

FIG. 4. Diagram for the decay of $\tau^{-} \rightarrow \nu_{\tau} K^{-} K_{1}(1)$, where $K_{1}(1)$ is the first pole of $K_{1}(1270)$. frame where the two meson system is at rest, and we assume that the quark spinors are at rest for the evaluation of the $Q_{\mu}$ matrix element in the same frame [19]. Then we have $\gamma^{0} \rightarrow 1, \gamma^{i} \gamma_{5} \rightarrow \sigma^{i}$ in terms of bispinors $\chi$, and after the spin angular momentum combination we end up with the following spinor matrix elements:

$$
\begin{aligned}
& Q_{0}=\left\langle\chi^{\prime}|1| \chi\right\rangle \equiv M_{0}, \\
& Q_{i}=\left\langle\chi^{\prime}\left|\sigma_{i}\right| \chi\right\rangle \equiv N_{i} .
\end{aligned}
$$

Denoting for simplicity,

$$
\bar{L}^{\mu \nu} \equiv \bar{\sum} \sum L^{\mu} L^{\nu \dagger}
$$

to obtain the $\tau$ width we must evaluate

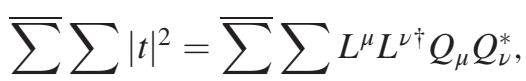

$$
\begin{aligned}
& =\bar{L}^{00} M_{0} M_{0}^{*}+\bar{L}^{0 i} M_{0} N_{i}^{*}+\bar{L}^{i 0} N_{i} M_{0}^{*} \\
& +\bar{L}^{i j} N_{i} N_{j}^{*} \text {, }
\end{aligned}
$$

with $\bar{L}^{\mu \nu}$ given by

$$
\begin{aligned}
\bar{\sum} \sum L^{\mu} L^{\nu \dagger}= & \frac{1}{m_{\nu} m_{\tau}}\left(p^{\prime \mu} p^{\nu}+p^{\prime \nu} p^{\mu}-g^{\mu \nu} p^{\prime} \cdot p\right. \\
& \left.+i \epsilon^{\alpha \mu \beta \nu} p_{\alpha}^{\prime} p_{\beta}\right),
\end{aligned}
$$

where $p, p^{\prime}$ are the momenta of the $\tau$ and $\nu_{\tau}$ respectively and we use the field normalization for fermions of Ref. [28]. The expression of the amplitudes in terms of the $M_{0}$ and $N_{i}$ functions was the main novelty of the work in [19]. This formalism has the advantage of filtering the $G$-parity contributions since the $M_{0}$ and $N_{i}$ operators act with defined $G$-parity as explained below.

From the work [19] we obtain the results for the $V V$, $J=1, J^{\prime}=1$ case,

$$
M_{0}=\frac{1}{\sqrt{3}} \frac{1}{4 \pi} \mathcal{C}\left(111 ; M, M^{\prime}, M+M^{\prime}\right),
$$

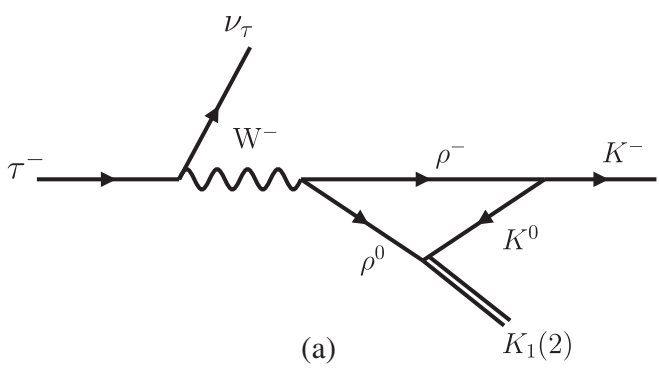

(a)

FIG. 5. Diagram for the decay of $\left.\tau^{-} \rightarrow \nu_{\tau} K^{-} K_{1}(2)\right)$, where $K_{1}(2)$ is the second pole of $K_{1}(1270)$.

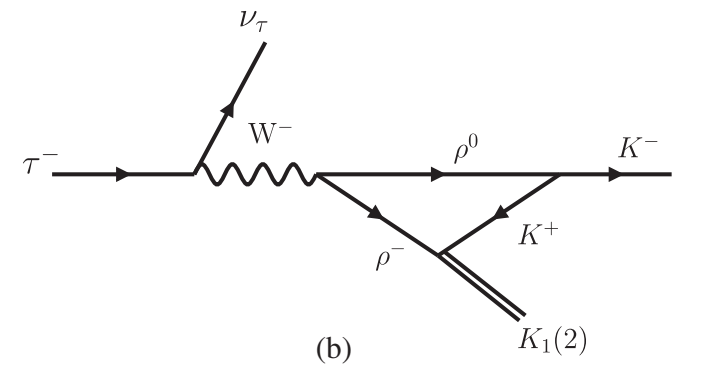

(b) 
TABLE IV. Signs resulting in the $M_{0}$, and $N_{\mu}$ amplitudes for $s$-wave by permuting the order of the mesons.

\begin{tabular}{lcccc}
\hline \hline & PP & PV & VP & VV \\
\hline$M_{0}$ & - & + & + & - \\
$N_{\mu}$ & + & - & - & + \\
\hline \hline
\end{tabular}

$$
\begin{aligned}
N_{\mu}= & \frac{1}{\sqrt{6}} \frac{1}{4 \pi}\left\{\delta_{M \mu}+2(-1)^{-\mu-M^{\prime}} \mathcal{C}(111 ; M,-\mu, M-\mu)\right. \\
& \left.\times \mathcal{C}\left(111 ; M^{\prime},-M-M^{\prime}+\mu,-M+\mu\right)\right\},
\end{aligned}
$$

where $M, M^{\prime}$ are the third components of $J, J^{\prime}$ respectively, and $\mu$ is the index of $N_{i}$ in spherical basis, with $\mathcal{C}(\cdots)$ a Clebsch-Gordan coefficient.

In [19], it was shown that the order in which the vector mesons are produced is essential to understand the $G$-parity symmetry of these reactions, which is given in Table IV. We note from Table IV that $M_{0}$ changes sign for VV, when exchanging the mesons, while for $N_{i}$ it is the same. This sign is essential for the conservation of $G$-parity in the reaction, as we shall see. On the other hand, at the quark level the primary $d \bar{u}$ state produced has $I_{3}=-1$ and hence $I=1$. The $G$-parity of a $q \bar{q}$ pair is given by $(-1)^{L+S+I}$. As we mentioned, $L=1, I=1$ and the spin of the state is 0 for the 1 operator and 1 for the $\sigma^{i}$ operator of Eq. (4). This means that the term $N_{i}$ proceeds with $G$-parity negative, while $M_{0}$ has $G$-parity positive. Since $\pi, h_{1}(1170)$, $h_{1}(1380), a_{1}(1260), \quad f_{1}(1285)$, and $b_{1}(1235)$ have $G$-parity,,,,,----++ respectively, then $\pi^{-} f_{1}(1285)$ and $\pi^{-} b_{1}(1235)$ proceed with the $N_{i}$ amplitude, while $\pi^{-} h_{1}(1170), \pi^{-} h_{1}(1380)$ and $\pi^{-} a_{1}(1260)$ will proceed with the $M_{0}$ term and there is no simultaneous contribution of the two terms in these reactions, implying that the crossed terms in Eq. (6) are zero. We shall see this analytically when evaluating explicitly the amplitudes for the processes of Figs. 1 and 3.

\section{Evaluation of the constant $\mathcal{C}$}

The global unknown constant $\mathcal{C}$ in Eq. (1) can be determined from the experimental ratio of $\tau \rightarrow \nu_{\tau} K^{* 0} K^{*-}$, using a similar method as in Ref. [13].

In the present work, the structure of $\sum \sum|t|^{2}$ for the $\tau$ decay into two vector mesons is taken from the results of [19] for this reaction. If we take the quantization axis along the direction of the neutrino in the $\tau^{-}$rest frame we find

$$
\begin{aligned}
\overline{\sum \sum|t|^{2}=} & \frac{\mathcal{C}^{2}}{m_{\tau} m_{\nu}}\left(\frac{1}{4 \pi}\right)^{2}\left[\left(E_{\tau} E_{\nu}+p^{2}\right) h_{i}^{2}\right. \\
& \left.+\frac{7}{2}\left(E_{\tau} E_{\nu}-\frac{1}{3} p^{2}\right) \bar{h}_{i}^{2}\right]
\end{aligned}
$$

where $p$ is the momentum of the $\tau$, or $\nu_{\tau}$, in the $K^{* 0} K^{*-}$ rest frame, given by
TABLE V. The $h_{i}$ and $\bar{h}_{i}$ coefficients for the different VV channels with the two vectors in $s$-wave.

\begin{tabular}{lcc}
\hline \hline channels & $h_{i}\left(\right.$ for $\left.M_{0}\right)$ & $\bar{h}_{i}\left(\right.$ for $\left.N_{\mu}\right)$ \\
\hline$K^{* 0} K^{*-}$ & 1 & 1 \\
$\rho^{-} \rho^{0}$ & $\sqrt{2}$ & 0 \\
\hline \hline
\end{tabular}

$$
p=p_{\nu}=p_{\tau}=\frac{\lambda^{1 / 2}\left(m_{\tau}^{2}, m_{\nu}^{2}, M_{\mathrm{inv}}^{2}\left(K^{* 0} K^{*-}\right)\right)}{2 M_{\mathrm{inv}}\left(K^{* 0} K^{*-}\right)},
$$

and $E_{\nu}=p, E_{\tau}=\sqrt{m_{\tau}^{2}+p^{2}}$. In Eq. (10) the coefficients $h_{i}$ and $\bar{h}_{i}$ account for the weights of the VV components for $M_{0}$ and $N_{\mu}$ respectively and their values are listed in Table V. Note that we are considering only the final s-wave production since, as explained in [19], because of the large vector masses, the expected momenta are very small.

The mass distribution is given by

$\frac{d \Gamma}{d M_{\mathrm{inv}}\left(K^{* 0} K^{*-}\right)}=\frac{2 m_{\tau} 2 m_{\nu}}{(2 \pi)^{3}} \frac{1}{4 m_{\tau}^{2}} p_{\nu}^{\prime} \tilde{p}_{K} \sum^{-} \sum|t|^{2}$,

where $\tilde{p}_{K}$ is the momentum of $K^{-}$in the $K^{* 0} K^{*-}$ rest frame given by

$$
\tilde{p}_{K}=\frac{\lambda^{1 / 2}\left(M_{\mathrm{inv}}^{2}\left(K^{* 0} K^{*-}\right), m_{K^{* 0}}^{2}, m_{K^{*-}}^{2}\right)}{2 M_{\mathrm{inv}}\left(K^{* 0} K^{*-}\right)},
$$

and $p_{\nu}^{\prime}$ the neutrino momentum in the $\tau$ rest frame

$$
p_{\nu}^{\prime}=\frac{\lambda^{1 / 2}\left(m_{\tau}^{2}, m_{\nu}^{2}, M_{\mathrm{inv}}^{2}\left(K^{* 0} K^{*-}\right)\right)}{2 m_{\tau}} .
$$

The experimental branching ratio of the $\tau \rightarrow \nu_{\tau} K^{* 0} K^{*-}$ decay was constructed in Ref. [13] from information in the PDG [5], with the result,

$$
\begin{aligned}
\mathcal{B}\left(\tau \rightarrow \nu_{\tau} K^{* 0} K^{*-}\right) & =\frac{1}{\Gamma_{\tau}} \Gamma\left(\tau \rightarrow \nu_{\tau} K^{* 0} K^{*-}\right) \\
& =(2.1 \pm 0.5) \times 10^{-3},
\end{aligned}
$$

from which we can evaluate the value of the constant $\mathcal{C}^{2}$,

$$
\frac{\mathcal{C}^{2}}{\Gamma_{\tau}}=\frac{\mathcal{B}\left(\tau \rightarrow \nu_{\tau} K^{* 0} K^{*-}\right)}{\int \frac{d \Gamma}{d M_{\mathrm{inv}}\left(K^{* 0} K^{*-}\right)} d M_{\mathrm{inv}}\left(K^{* 0} K^{*-}\right)}=5.0 \times 10^{-4}\left[\mathrm{MeV}^{-1}\right] .
$$

Note that the $\tau$ decay into $\nu_{\tau} K^{* 0} K^{*-}$ can only proceed because of the finite width of the $K^{*}$, since otherwise there would be no available phase space for infinitely narrow $K^{*}$. Hence it is crucial to fold the width with a realistic spectral function of the $K^{*}$ meson, (see Eq. (9) in [13]). Note that, in Ref. [13], the structure of $\bar{\sum} \sum|t|^{2}$ was assumed to 
proceed with the dominant term $E_{\tau} E_{\nu}-\frac{p^{2}}{3}$ of Eq. (10) alone, and hence a somewhat different $\mathcal{C}$ constant was obtained.

\section{Evaluation of the triangle diagram}

In order to evaluate the triangle loops of Fig. 1, we need first the $K^{*} \rightarrow K \pi$ vertex obtained from the VPP Lagrangian

$$
\mathcal{L}_{\mathrm{VPP}}=-i g\left\langle\left[P, \partial_{\mu} P\right] V^{\mu}\right\rangle,
$$

with the coupling $g=4.31$ [13], $P$ and $V$ the SU(3) matrices of the pseudoscalar and vector mesons, by means of which we find

$$
\begin{gathered}
t_{K^{* 0} \rightarrow \pi^{-} K^{+}}=(2 \boldsymbol{k}+\boldsymbol{q}) \cdot \boldsymbol{\epsilon} g, \\
t_{K^{*-} \rightarrow \pi^{-} K^{0}}=-(2 \boldsymbol{k}+\boldsymbol{q}) \cdot \boldsymbol{\epsilon} g .
\end{gathered}
$$

We can see that for this vertex we find a relative minus sign from Fig. 1(a) to Fig. 1(b). We find it convenient to take the $z$ direction along the momentum $\boldsymbol{k}$ of the final pion (see Fig. 2). Indeed, in the $\pi A$ rest frame, where we evaluate the amplitude, $\boldsymbol{P}=0$. The vertex $K^{*} \rightarrow K \pi$ is of the type $\boldsymbol{\epsilon} \cdot(\boldsymbol{k}+\boldsymbol{q}+\boldsymbol{k}){ }^{1}$ On the other hand, the $\boldsymbol{q}$ integration $\int d \boldsymbol{q}^{i}(2 \boldsymbol{k}+\boldsymbol{q}) \cdots$ of the triangle loop must necessarily give something proportional to $\boldsymbol{k}$, which is the only nonintegrated vector in the loop integral. Then $\int d \boldsymbol{q}^{i}(2 \boldsymbol{k}+\boldsymbol{q}) \cdots=A \boldsymbol{k}$ and contracting with $\boldsymbol{k}$ gives $\boldsymbol{k} \int d \boldsymbol{q}^{i}\left(2+\boldsymbol{q} \cdot \boldsymbol{k} / \boldsymbol{k}^{2}\right) \cdots$. Hence, we have an effective vertex of the type $\boldsymbol{\epsilon} \cdot \boldsymbol{k}$. If the $z$ direction is chosen along $\boldsymbol{k}$, this selects only the $\boldsymbol{\epsilon}_{z}$ component ( $\boldsymbol{\epsilon}_{0}$ in spherical basis) and $\boldsymbol{\epsilon} \cdot \boldsymbol{k}=|\boldsymbol{k}|=k$. This also means that only $M=0$, for Eqs. (8) and (9), contributes in the loop and this allows us to calculate trivially the $M_{0}$ and $N_{\mu}$ amplitudes in that frame.

\section{Evaluation of $M_{0}$}

For $K^{* 0}(M)$ and $K^{*-}\left(M^{\prime}\right)$ of Fig. 1(a) and $M=0$, we get from Eq. (8)

$$
M_{0} \rightarrow \frac{1}{\sqrt{3}} \frac{1}{4 \pi} \mathcal{C}\left(111 ; 0, M^{\prime}, M^{\prime}\right) .
$$

On the other hand, in Fig. 1(b) we will have $M^{\prime}$ of $K^{*-}$ equal to zero and then

$$
\begin{aligned}
M_{0} & \rightarrow \frac{1}{\sqrt{3}} \frac{1}{4 \pi} \mathcal{C}(111 ; M, 0, M) \\
& =(-1) \frac{1}{\sqrt{3}} \frac{1}{4 \pi} \mathcal{C}(111 ; 0, M, M) .
\end{aligned}
$$

\footnotetext{
${ }^{1}$ Since in the triangle diagram the $K^{* 0} K^{*-}$ intermediate states have a small momentum compared to the $K^{*}$ mass [13], we neglect the $\boldsymbol{\epsilon}^{0}$ component, which was found in [29] to be an excellent approximation in such a case.
}

We can see that the $M_{0}$ changes sign from Fig. 1(a) to Fig. 1(b). From the sign of Eqs. (18) and (19) and this latter sign, we can see that the global sign is the same for these two diagrams.

Finally, in order to evaluate the final amplitude of the loop diagram we need the vertex $A \rightarrow V P$, which is of the type [11]

$$
g_{A, V P} \boldsymbol{\epsilon}_{V} \cdot \boldsymbol{\epsilon}_{A}
$$

with $\boldsymbol{\epsilon}_{V}, \boldsymbol{\epsilon}_{A}$, the polarization vector of the vector and axialvector resonances. Note that the $\boldsymbol{\epsilon}_{V} \cdot \boldsymbol{\epsilon}_{A}$ structure is indeed an approximation of the more general possible like the one in Eq. (11) in Ref. [22]. However the later reduces to ours considering that the $\epsilon^{0}$ components usually give a small contribution of the order of $|\vec{p} / M|^{2}$ (see for instance Ref. [11,29] and that the couplings are evaluated at the axial poles and dominates the axials on-shell. The evaluation in [11] is done for pseudoscalar and vectors mesons on shell, while we have them in a loop here. Yet, the proximity to the triangle singularity of the mechanism considered makes the on shell contribution of the particles more important and we can then use the on shell information for the vertices.

The couplings of the axials to pseudoscalar and vector are given in [11] in terms of well defined $G$-parity PV states (see Tables I-III). In order to relate those couplings to the charge basis PV states that we are using, we need to write our states for the axial vector mesons in terms of their vector pseudoscalar components forming states of welldefined $C$ and $G$ parity:

$$
\begin{aligned}
|I=0, C=-\rangle & =\frac{1}{\sqrt{2}}\left(\left|\bar{K}^{*} K\right\rangle_{I=0}-\left|K^{*} \bar{K}\right\rangle_{I=0}\right) \\
& =+\frac{1}{2}\left(K^{*+} K^{-}+K^{* 0} \bar{K}^{0}+K^{*-} K^{+}+\bar{K}^{* 0} K^{0}\right) \\
|I=0, C=+\rangle & =\frac{1}{\sqrt{2}}\left(\left|\bar{K}^{*} K\right\rangle_{I=0}+\left|K^{*} \bar{K}\right\rangle_{I=0}\right) \\
& =-\frac{1}{2}\left(K^{*+} K^{-}+K^{* 0} \bar{K}^{0}-K^{*-} K^{+}-\bar{K}^{* 0} K^{0}\right),
\end{aligned}
$$

$$
\begin{aligned}
|I=1, C=-\rangle & =\frac{1}{\sqrt{2}}\left(\left|\bar{K}^{*} K\right\rangle_{I=1}+\left|K^{*} \bar{K}\right\rangle_{I=1}\right) \\
& =-\frac{1}{2}\left(K^{*+} K^{-}-K^{* 0} \bar{K}^{0}+K^{*-} K^{+}-\bar{K}^{* 0} K^{0}\right) \\
|I=1, C=+\rangle & =\frac{1}{\sqrt{2}}\left(\left|\bar{K}^{*} K\right\rangle_{I=1}-\left|K^{*} \bar{K}\right\rangle_{I=1}\right) \\
& =+\frac{1}{2}\left(K^{*+} K^{-}-K^{* 0} \bar{K}^{0}-K^{*-} K^{+}+\bar{K}^{* 0} K^{0}\right) .
\end{aligned}
$$


Since $G=(-1)^{I} C$, we need from the sum of Figs. 1(a) and $1(\mathrm{~b})$, the following combinations.

$\begin{array}{ll}I=0, C=-, G=-, & g_{A, K^{*-} K^{+}}+g_{A, K^{* 0} \bar{K}^{0}}=+g_{A, K^{*} \bar{K}}, \\ I=0, C=+, G=+, & g_{A, K^{*-} K^{+}}+g_{A, K^{* 0} \bar{K}^{0}}=0, \\ I=1, C=-, G=+, & g_{A, K^{*-} K^{+}}+g_{A, K^{* 0} \bar{K}^{0}}=0, \\ I=1, C=+, G=-, & g_{A, K^{*} K^{+}}+g_{A, K^{* 0} \bar{K}^{0}}=-g_{A, K^{*} \bar{K}} .\end{array}$

Note that Eq. (22) implies that the $M^{\prime}$ third component of Eq. (20) becomes the $M_{A}$ third component of the axial vector $A$. Then the $M_{0}$ contribution to $h_{1}$ and $a_{1}$ from the $K^{*} \bar{K}^{*}$ loop becomes

$$
t_{M_{0}}=\mathcal{C} g k h_{K^{* 0} K^{*-}} \mathcal{C}\left(111 ; 0, M_{A}, M_{A}\right)( \pm 1) g_{A, K^{*}} t_{L}\left(K^{*} \bar{K}^{*}\right),
$$

with the,+- sign for $h_{1}$ and $a_{1}$ production, respectively, and

$$
\begin{aligned}
t_{L}= & \int \frac{d^{3} q}{(2 \pi)^{3}} \frac{2+\boldsymbol{k} \cdot \boldsymbol{q} /|\boldsymbol{k}|^{2}}{8 \omega_{1} \omega_{2} \omega_{3}} \frac{1}{k^{0}-\omega_{3}-\omega_{1}+i \epsilon} \\
& \times \frac{1}{P^{0}-\omega_{1}-\omega_{2}+i \epsilon} \\
& \times \frac{2 P^{0} \omega_{2}+2 k^{0} \omega_{3}-2\left(\omega_{2}+\omega_{3}\right)\left(\omega_{2}+\omega_{3}+\omega_{1}\right)}{\left(P^{0}-\omega_{2}-\omega_{3}-k^{0}+i \epsilon\right)\left(P^{0}+\omega_{2}+\omega_{3}-k^{0}-i \epsilon\right)},
\end{aligned}
$$

is the triangle loop function which appears after an analytical calculation of the $q^{0}$ integral [30]. For the case of the diagrams in Fig. 1(a) the states 1, 2, 3 correspond to $K^{* 0}, K^{*-}, K^{+}$, respectively. In Eq. (27) $\omega_{1}=\sqrt{\boldsymbol{q}^{2}+m_{1}^{2}}$, $\omega_{2}=\sqrt{\boldsymbol{q}^{2}+m_{2}^{2}}$ and $\omega_{3}=\sqrt{(\boldsymbol{q}+\boldsymbol{k})^{2}+m_{3}^{2}}$ are the energies of the 1,2 and 3 states in the loop respectively, $P^{0}=M_{\text {inv }}\left(\pi^{-} A\right)$, and

$$
\begin{aligned}
& k^{0}=\frac{M_{\mathrm{inv}}^{2}\left(\pi^{-} A\right)+m_{\pi}^{2}-m_{A}^{2}}{2 M_{\mathrm{inv}}\left(\pi^{-} A\right)}, \\
& k=\frac{\lambda^{1 / 2}\left(M_{\mathrm{inv}}^{2}\left(\pi^{-} A\right), m_{\pi}^{2}, m_{A}^{2}\right)}{2 M_{\mathrm{inv}}\left(\pi^{-} A\right)} .
\end{aligned}
$$

We also account for the $K^{* 0}, K^{*-}$ widths by replacing $\omega_{1} \rightarrow \omega_{1}-i \frac{\Gamma_{K^{*}}}{2}, \quad \omega_{2} \rightarrow \omega_{2}-i \frac{\Gamma_{K^{*}}}{2}$ in the propagators involving $\omega_{K^{*}}$ in the actual calculation. Similarly, we can get the triangle amplitude for Fig. 1(b) case.

\section{Evaluation of $N_{i}$}

In spherical basis, $N_{i} \rightarrow N_{\mu}(\mu=0, \pm 1)$, is given by Eq. (9). Once again we choose now $\boldsymbol{k}$ in the $z$ direction and thus force $M=0$. On the other hand, as we did before, $M^{\prime}$ becomes $M_{A}$, the axial vector polarization, since the $s$-wave coupling of $A \rightarrow P V\left(\boldsymbol{\epsilon}_{A} \cdot \boldsymbol{\epsilon}\right)$ implies the same $A$ and $V$ polarization. Then Eq. (9) becomes

$$
\begin{aligned}
N_{\mu}= & \frac{1}{\sqrt{6}} \frac{1}{4 \pi}\left\{\delta_{\mu 0}+2(-1)^{-\mu-M_{A}} \mathcal{C}(111 ; 0,-\mu,-\mu)\right. \\
& \left.\times \mathcal{C}\left(111 ; M_{A},-M_{A}+\mu, \mu\right)\right\} .
\end{aligned}
$$

Contrary to what happens with the $M_{0}$ component, we can see that $N_{\mu}$ does not change sign when we exchange $K^{* 0} K^{*-} \rightarrow K^{*-} K^{* 0}$ in the loop of Fig. 1(a) and Fig. 1(b). Then, considering the different sign in the vertex $K^{*} \rightarrow K \pi$ of Eqs. (18) and (19), we get the combination $g_{A, K^{*-} K^{+}}-g_{A, K^{* 0} \bar{K}^{0}}$, which in view of Eqs. (23) and (24) provides,

$I=0, C=-, G=-, \quad g_{A, K^{*-} K^{+}}-g_{A, K^{* 0} \bar{K}^{0}}=0$,

$I=0, C=+, G=+, \quad g_{A, K^{*-} K^{+}}-g_{A, K^{* 0} \bar{K}^{0}}=g_{A, K^{*} \bar{K}}$,

$I=1, C=-, G=+, \quad g_{A, K^{*-} K^{+}}-g_{A, K^{* 0} \bar{K}^{0}}=-g_{A, K^{*} \bar{K}}$.

$I=1, C=+, G=-, \quad g_{A, K^{*-} K^{+}}-g_{A, K^{* 0} \bar{K}^{0}}=0$.

This shows explicitly that with $G$-parity positive axials only the $N_{i}$ term contributes, as we saw at the beginning at the quark level, while for $G$ negative axials only the $M_{0}$ term contributes.

From Eq. (30) we can calculate the $N_{i}$ components in the Cartesian basis and we find

$N_{1}=\frac{1}{\sqrt{2}}\left(N_{-1}-N_{+1}\right)=\frac{1}{\sqrt{2}} \begin{cases}-\frac{1}{\sqrt{6}} \frac{1}{4 \pi}, & \left(M_{A}=1\right) \\ 0, & \left(M_{A}=0\right) \\ \frac{1}{\sqrt{6}} \frac{1}{4 \pi}, & \left(M_{A}=-1\right)\end{cases}$

$N_{2}=\frac{i}{\sqrt{2}}\left(N_{-1}+N_{+1}\right)=\frac{1}{\sqrt{2}} \begin{cases}\frac{1}{\sqrt{6}} \frac{1}{4 \pi}, & \left(M_{A}=1\right) \\ \frac{2}{\sqrt{6}} \frac{1}{4 \pi}, & \left(M_{A}=0\right) \\ \frac{1}{\sqrt{6}} \frac{1}{4 \pi}, & \left(M_{A}=-1\right)\end{cases}$

$$
N_{3}=N_{0}=\frac{1}{\sqrt{6}} \frac{1}{4 \pi}, \quad\left(\text { for any } M_{A}\right)
$$

\section{E. Incorporation of intermediate $\rho \rho$ states}

For the production of negative $G$-parity axial vector mesons, we must also consider the $\rho^{0} \rho^{-}$diagrams of Fig. 3 in addition to Fig. 1. For this we need the $h_{i}$ coefficients of 
Table V. Recall that in this case only the $M_{0}$ term contributes. Next we need the $\rho^{-} \rightarrow \pi^{-} \pi^{0}, \rho^{0} \rightarrow \pi^{-} \pi^{+}$ vertices obtained from the Lagrangian in Eq. (17),

$$
\begin{gathered}
t_{\rho^{-} \rightarrow \pi^{-} \pi^{0}}=\sqrt{2} g(2 \boldsymbol{k}+\boldsymbol{q}) \cdot \boldsymbol{\epsilon}, \\
t_{\rho^{0} \rightarrow \pi^{-} \pi^{+}}=-\sqrt{2} g(2 \boldsymbol{k}+\boldsymbol{q}) \cdot \boldsymbol{\epsilon} .
\end{gathered}
$$

Since $M_{0}$ changes sign from $\rho^{-} \rho^{0}$ to $\rho^{0} \rho^{-}$production (see Table IV), this sign and the relative one of Eqs. (35) and (36) cancel and we get the factor in the sum of the loops

$$
g_{A, \rho^{0} \pi^{0}}+g_{A, \rho^{-} \pi^{+}}
$$

To relate these couplings in charge basis to the coupling of $A$ to $\rho \pi$ in isospin basis, evaluated in [11], we recall that the isospin multiplets are $\left(-\pi^{+}, \pi^{0}, \pi^{-}\right),\left(-\rho^{+}, \rho^{0}, \rho^{-}\right)$. Then we have

$$
\begin{aligned}
& \left|\rho \rho, I=0, I_{3}=0\right\rangle=-\frac{1}{\sqrt{3}}\left(\rho^{+} \pi^{-}+\rho^{-} \pi^{+}+\rho^{0} \pi^{0}\right), \\
& \left|\rho \rho, I=1, I_{3}=0\right\rangle=\frac{1}{\sqrt{2}}\left(\rho^{-} \pi^{+}-\rho^{+} \pi^{-}\right) .
\end{aligned}
$$

Then

$$
\begin{array}{ll}
g_{A, \rho^{0} \pi^{0}}+g_{A, \rho^{-} \pi^{+}}=-\frac{2}{\sqrt{3}} g_{A, \rho \pi}, & \text { for } I=0\left(h_{1}\right) \\
g_{A, \rho^{0} \pi^{0}}+g_{A, \rho^{-} \pi^{+}}=\frac{1}{\sqrt{2}} g_{A, \rho \pi}, & \text { for } I=1\left(a_{1}\right)
\end{array} .
$$

The $\rho \pi$ channel only contributes to these two states that have negative $G$-parity.

Thus, in order to account for the coherent sum of $K^{*-} K^{* 0}$ and $\rho^{-} \rho^{0}$ we can use $t_{M_{0}}$ of Eq. (26) but performing the following substitution,

$g_{A, K^{*} \bar{K}} t_{L}\left(K^{*} \bar{K}^{*}\right) \rightarrow g_{A, K^{*} \bar{K}} t_{L}\left(K^{*} \bar{K}^{*}\right)+2 D g_{A, \rho \pi} t_{L}(\rho \rho)$,

with

$$
D= \begin{cases}-\frac{2}{\sqrt{3}}, & \text { for } I=0\left(h_{1}\right) \\ \frac{1}{\sqrt{2}}, & \text { for } I=1\left(a_{1}\right)\end{cases}
$$

Next we must perform the sum of Eq. (6) independently, $\bar{L}^{00} M_{0} M_{0}^{*}$ for negative $G$-parity $A$ states and $\bar{L}^{i j} N_{i} N_{j}^{*}$ for positive $G$-parity $A$ states. By using Eq. (7) and Eqs. (20), (32)-(34) and summing over the $M_{A}$ components we obtain: (a) $G$-parity positive axial states:

$$
\begin{aligned}
& \bar{\sum} \sum|t|^{2}=\frac{\mathcal{C}^{2}}{m_{\tau} m_{\nu}} \frac{1}{(4 \pi)^{2}} \frac{7}{6}\left(E_{\tau} E_{\nu}-\frac{1}{3} p^{2}\right) \\
& \times g^{2} k^{2}\left|g_{A, K^{*} \bar{K}}\right|^{2}\left|t_{L}\left(K^{*} \bar{K}^{*}\right)\right|^{2},
\end{aligned}
$$

(b) $G$-parity negative axial states:

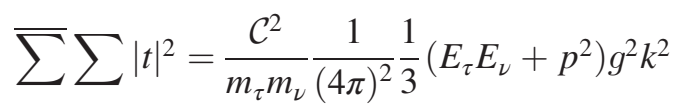

$$
\begin{aligned}
& \times\left|g_{A, K^{*} \bar{K}} t_{L}\left(K^{*} \bar{K}^{*}\right)+2 D g_{A, \rho \pi} t_{L}(\rho \rho)\right|^{2} .
\end{aligned}
$$

We should note that the $\epsilon^{\alpha \mu \beta \nu} p_{\alpha}^{\prime} p_{\beta}$ term of Eq. (7) does not contribute in $M_{0}$ since $\mu=0$ and $p_{\alpha}^{\prime} p_{\beta}\left(p_{\tau} p_{\nu}\right)$ will be spatial and $\boldsymbol{p}_{\tau} \boldsymbol{p}_{\nu}$ are the same in the frame we work. For $N_{i}$, $\alpha$ or $\beta$ must be zero and we have just one vector $\boldsymbol{p}_{\nu}$ that cancels in the phase space integration. For the same reason, the term with $p_{\nu i} p_{\nu j}$ becomes $\frac{1}{3} p_{\nu}^{2} \delta_{i j}$ upon integration over phase space.

$$
\text { F. } \tau^{-} \rightarrow \nu_{\tau} K^{-} K_{1}(1270)
$$

In [11] two states corresponding to $K_{1}$ were found and the pole positions were refined in [25], one of them at $1195 \mathrm{MeV}$ coupling mostly to $K^{*} \pi$, and another one at $1284 \mathrm{MeV}$ coupling mostly to $\rho K$. Proceeding analogously to the previous cases, the terms that go like $L^{0 i}$, of $\epsilon^{\alpha \mu \beta \nu}$ cancel again in the integration over phase space, and we obtain:

(a) $K_{1}(1)$ state:

$$
\begin{aligned}
\overline{\sum \sum|t|^{2}=} & \frac{\mathcal{C}^{2}}{m_{\tau} m_{\nu}} \frac{1}{(4 \pi)^{2}} g^{2} k^{2}\left|t_{L}\left(K^{*} \bar{K}^{*}\right)\right|^{2} \frac{1}{3}\left|g_{K_{1}, K^{*} \pi}\right|^{2} \\
& \times \frac{1}{2}\left[\frac{1}{3}\left(E_{\tau} E_{\nu}+p^{2}\right)+\frac{7}{6}\left(E_{\tau} E_{\nu}-\frac{1}{3} p^{2}\right)\right],
\end{aligned}
$$

(b) $K_{1}(2)$ state:

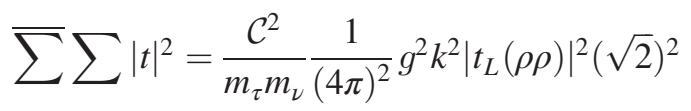

$$
\begin{aligned}
& \times \frac{1}{3}\left(E_{\tau} E_{\nu}+p^{2}\right) \frac{4}{3}\left|g_{K_{1}, \rho K}\right|^{2} \text {. }
\end{aligned}
$$

For $\tau^{-} \rightarrow \nu_{\tau} \pi^{-} A$ decay, the differential mass distribution for $M_{\text {inv }}\left(\pi^{-} A\right)$ is given by

$$
\frac{1}{\Gamma_{\tau}} \frac{d \Gamma}{d M_{\mathrm{inv}}\left(\pi^{-} A\right)}=\frac{1}{\Gamma_{\tau}} \frac{1}{(2 \pi)^{3}} \frac{2 m_{\tau} 2 m_{\nu}}{4 m_{\tau}^{2}} p_{\nu} \tilde{p}_{\pi} \bar{\sum} \sum|t|^{2},
$$




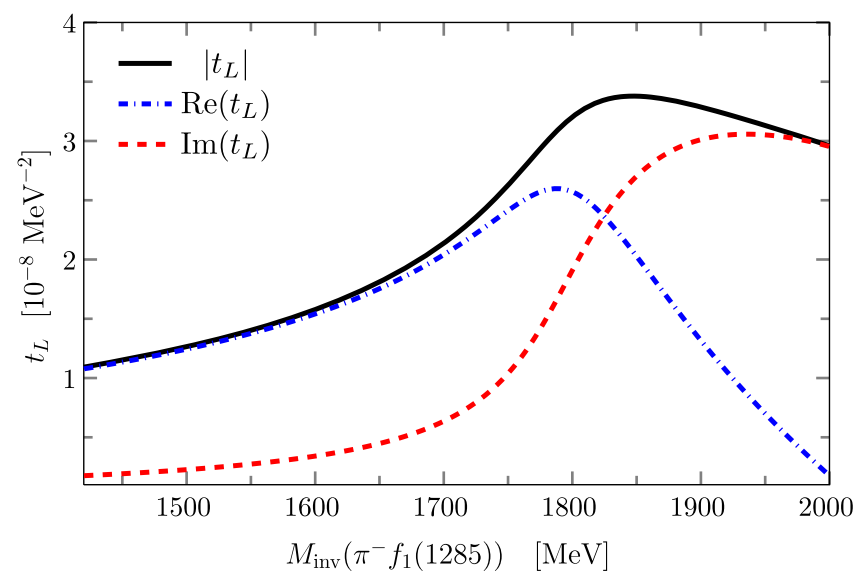

FIG. 6. Triangle amplitude $\operatorname{Re}\left(t_{L}\right), \operatorname{Im}\left(t_{L}\right)$ and $\left|t_{L}\right|$ for $\tau^{-} \rightarrow$ $\nu_{\tau} \pi f_{1}(1285)$ decay, taking $M_{\text {inv }}\left(f_{1}\right)=1229.5 \mathrm{MeV}$.

with

$$
\begin{aligned}
& p_{\nu}=\frac{\lambda^{1 / 2}\left(m_{\tau}^{2}, m_{\nu}^{2}, M_{\mathrm{inv}}^{2}\left(\pi^{-} A\right)\right)}{2 m_{\tau}}, \\
& \tilde{p}_{\pi}=\frac{\lambda^{1 / 2}\left(M_{\mathrm{inv}}^{2}\left(\pi^{-} A\right), m_{\pi}^{2}, m_{A}^{2}\right)}{2 M_{\mathrm{inv}}\left(\pi^{-} A\right)},
\end{aligned}
$$

and $p$ is the momentum of the $\tau$, or $\nu_{\tau}$, in the $\pi^{-} A$ rest frame, given by

$$
\tilde{p}=\frac{\lambda^{1 / 2}\left(m_{\tau}^{2}, m_{\nu}^{2}, M_{\mathrm{inv}}^{2}\left(\pi^{-} A\right)\right)}{2 M_{\mathrm{inv}}\left(\pi^{-} A\right)} .
$$

Similarly, for the $\tau^{-} \rightarrow \nu_{\tau} K^{-} K_{1}(1270)$ decay, we can get the differential mass distribution for $M_{\text {inv }}\left(K^{-} K_{1}(1270)\right)$.

Note that the term $m_{\tau} m_{\nu}$ in the numerator of Eq. (62) cancels the same factor in the denominator of Eqs. (42)-(45). In Eq. (62) we have the same factor $\frac{C^{2}}{\Gamma_{\tau}}$ from Eq. (16) and thus we can provide absolute values for the mass distributions.

\section{RESULTS}

First we show in Fig. 6 the triangle loop in Eq. (27), for the $\tau^{-} \rightarrow \nu_{\tau} \pi^{-} f_{1}(1285)$ case, as a function of the $\pi^{-} f_{1}$ invariant mass, $M_{\text {inv }}\left(\pi^{-} f_{1}\right)$. Note that there is a large increase of the strength at around the region of interest at the present work, $M_{\text {inv }}\left(\pi^{-} f_{1}\right)=m_{\tau}=1777 \mathrm{MeV}$, which will push the invariant mass distributions for the decays considered in the present work to the higher energy region of the spectrum, as we will see below. As already discussed in [13] the origin of this increase is twofold: first because of the presence of a nearby triangular singularity and, second, because of the presence of the $K^{*} \bar{K}^{*}$ threshold. Both effects are implicitly properly taken into account in the evaluation of the triangle loop in the present work. In Fig. 7 we see the triangle loop for $\rho \rho \pi$ as internal lines, for the $h_{1}(1170)$ in the final state. In this case we see that the enhancement is smaller because there is no a nearby singularity but some strength is visible from the $\rho \rho$ threshold.

In Figs. 8-13 we show the pseudoscalar-axial, $P A$, invariant mass distributions of the different $\tau^{-} \rightarrow \nu_{\tau} P A$ decays. We have considered the finite width of the axial resonances by folding the invariant mass distributions with the corresponding axial meson spectral function:

$\frac{d \Gamma_{\tau \rightarrow \nu_{\tau} P A}}{d M_{\mathrm{inv}}(P A)}=\frac{1}{N} \int_{\left(M_{A}-2 \Gamma_{A}\right)^{2}}^{\left(M_{A}+2 \Gamma_{A}\right)^{2}} d m^{2} \operatorname{Im} D(m) \frac{d \Gamma(m)}{d M_{\mathrm{inv}}(P A)}$,

where $D(m)$ is the axial-vector propagator,

$$
\begin{aligned}
D(m) & =\frac{1}{m^{2}-M_{A}^{2}+i \Gamma_{A} m_{A}}, \\
N & =\int_{\left(M_{A}-2 \Gamma_{A}\right)^{2}}^{\left(M_{A}+2 \Gamma_{A}\right)^{2}} d m^{2} \operatorname{Im} D(m),
\end{aligned}
$$

This folding is particularly relevant for the decays into $K_{1}$ because of the little and null available phase space for the

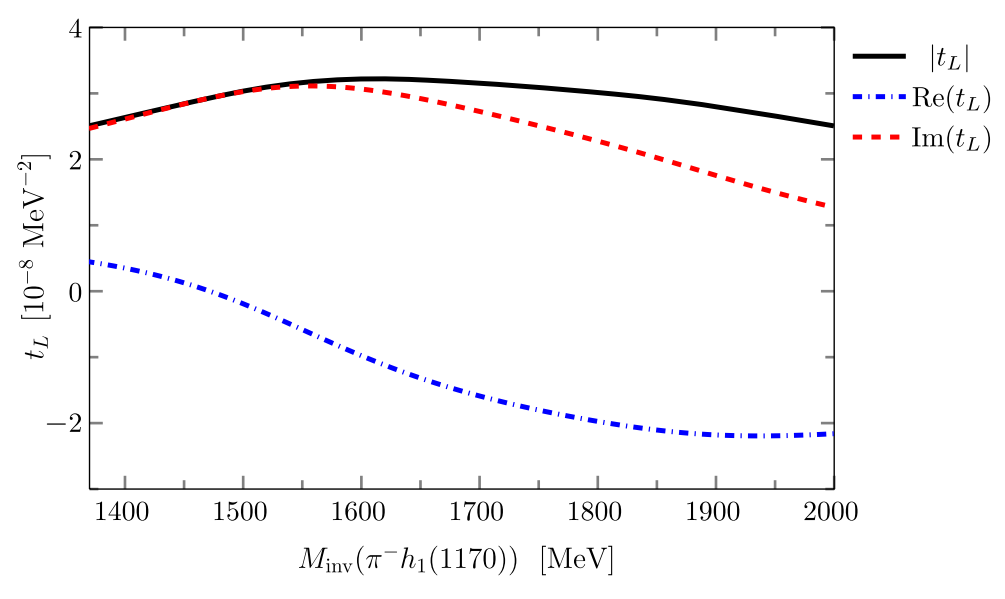

FIG. 7. Same as Fig. 6 for $\tau^{-} \rightarrow \nu_{\tau} \pi h_{1}(1170)$ decay. 


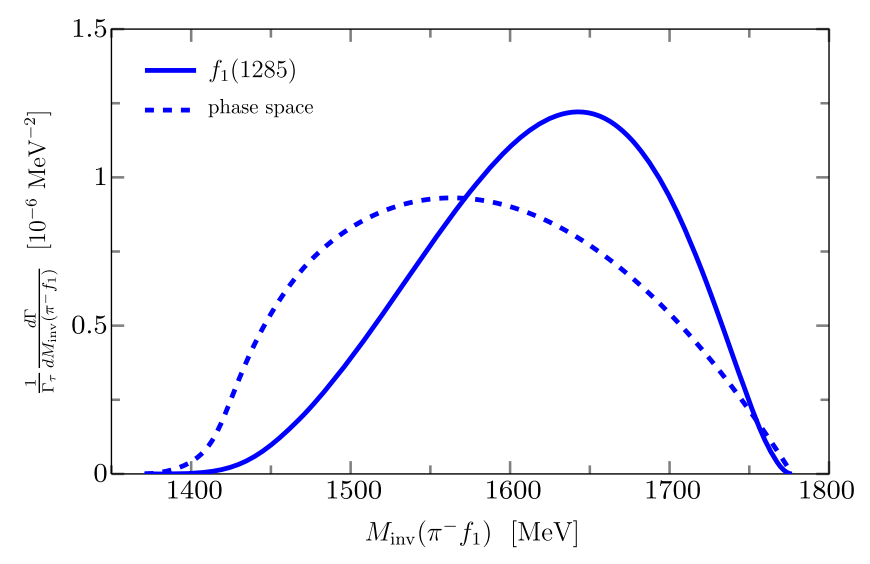

FIG. 8. Mass distribution for $\tau^{-} \rightarrow \nu_{\tau} \pi^{-} f_{1}(1285)$ decay.

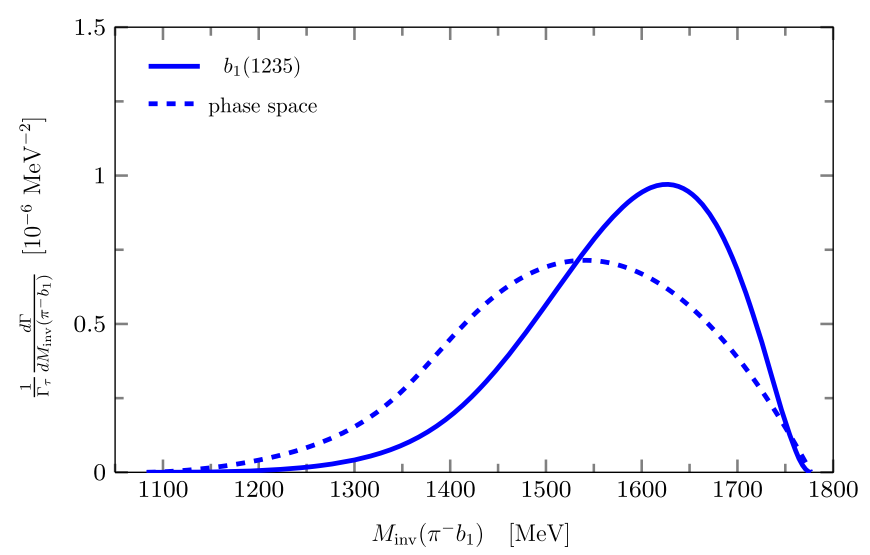

FIG. 9. Mass distribution for $\tau^{-} \rightarrow \nu_{\tau} \pi^{-} b_{1}(1235)$ decay.

$K_{1}(1)$ and $K_{1}(2)$ respectively. Actually $K_{1}(2)$ can only proceed because of its tail.

In Figs. 8-13 we also plot (dashed line) the phase space distribution normalized to the area below the full calculation. We can see that the strengths of the full calculations are moved to the higher mass of the spectrum, as a consequence of the particular increase of strength at these

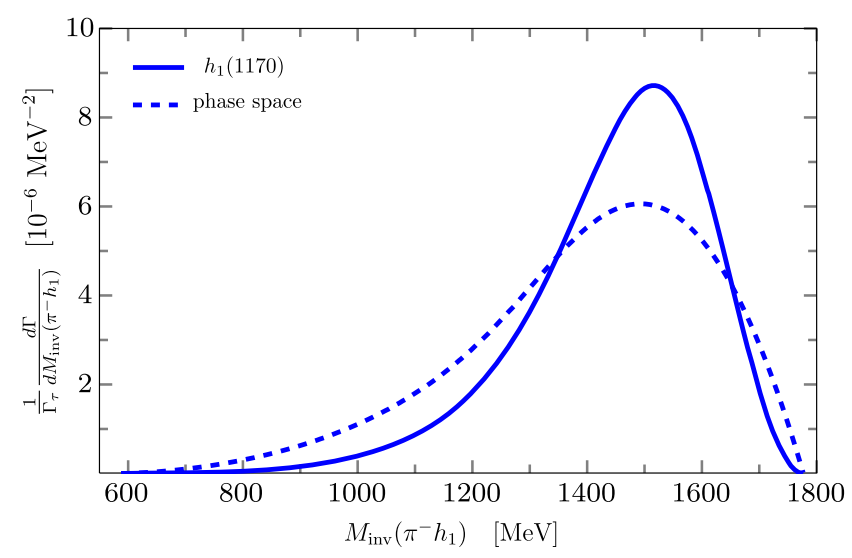

FIG. 10. Mass distribution for $\tau^{-} \rightarrow \nu_{\tau} \pi^{-} h_{1}(1170)$ decay.

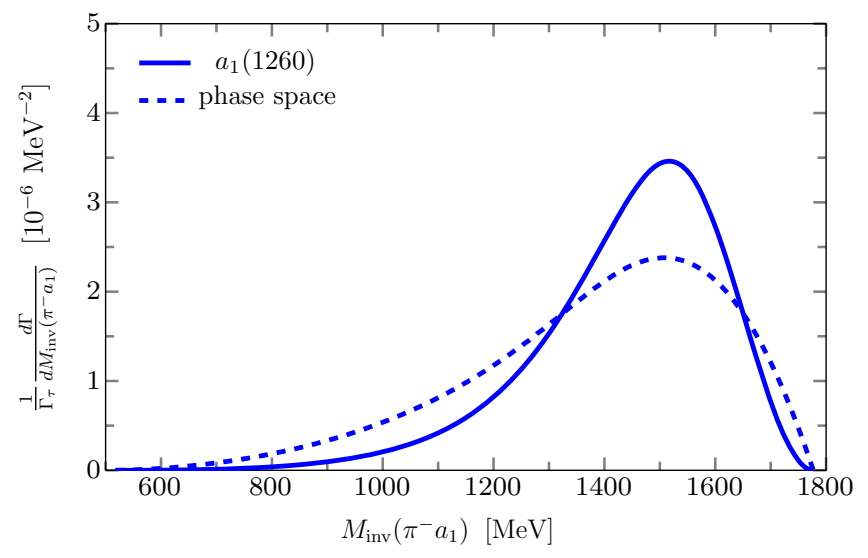

FIG. 11. Mass distribution for $\tau^{-} \rightarrow \nu_{\tau} \pi^{-} a_{1}(1260)$ decay.

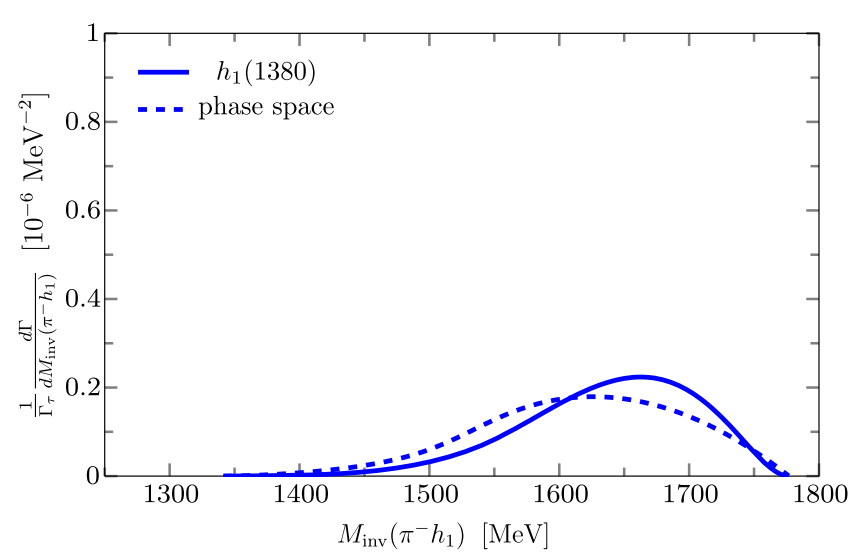

FIG. 12. Mass distribution for $\tau^{-} \rightarrow \nu_{\tau} \pi^{-} h_{1}(1380)$ decay.

energies of the triangle loop function. Figure 14 for $K_{1}(2)$ production is an exception tied to the fact that only the tail of the resonance allows its production.

In Table VI we show the integrated branching ratios for the different $\tau \rightarrow \nu_{\tau} P A$ decay channels. In Ref. [13] a careful error analysis was performed for the $f_{1}(1285)$ and an error of about $40 \%$ was obtained. For the present

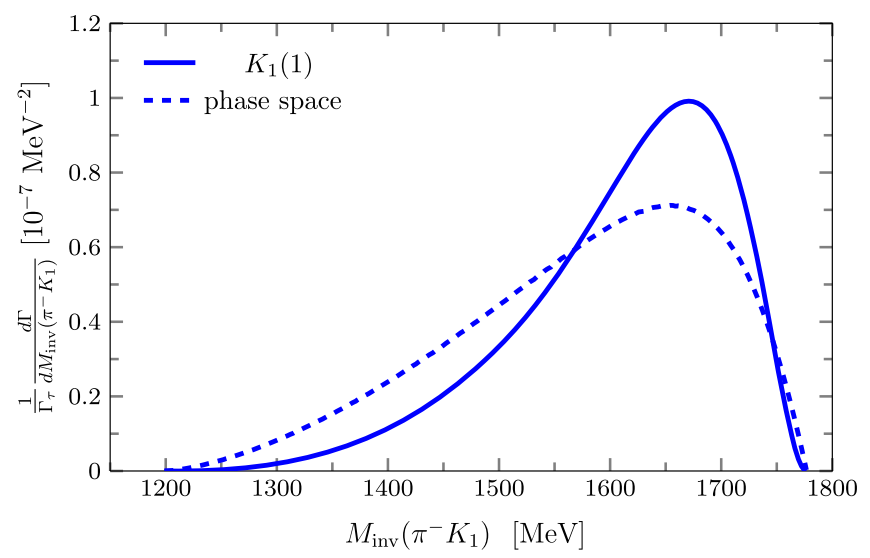

FIG. 13. Mass distribution for $\tau^{-} \rightarrow \nu_{\tau} K^{-} K_{1}(1)$ decay. 


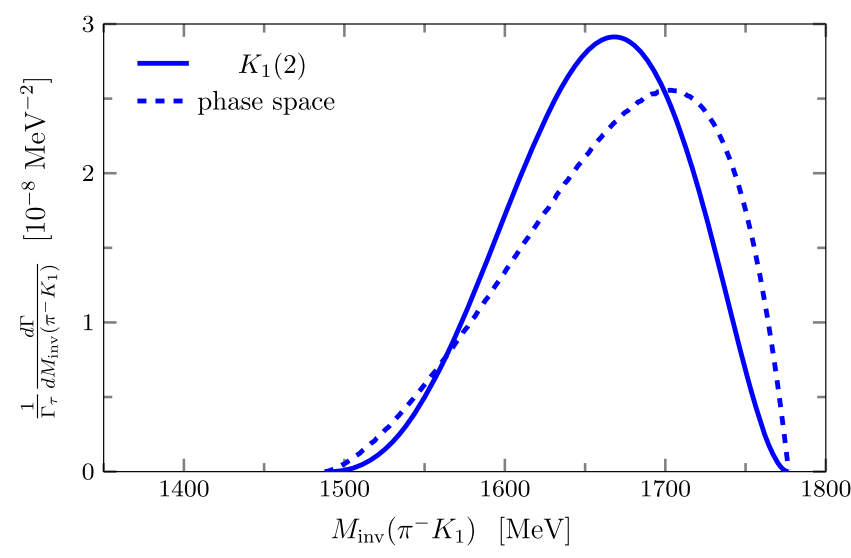

FIG. 14. Mass distribution for $\tau^{-} \rightarrow \nu_{\tau} K^{-} K_{1}(2)$ decay.

TABLE VI. The branching ratios for $\tau \rightarrow \pi^{-} A, K^{-} K_{1}$ decays.

\begin{tabular}{lc}
\hline \hline & $\mathcal{B}$ \\
\hline$h_{1}(1170)$ & $3.1 \times 10^{-3}$ \\
$a_{1}(1260)$ & $1.3 \times 10^{-3}$ \\
$b_{1}(1235)$ & $2.4 \times 10^{-4}$ \\
$f_{1}(1285)$ & $2.4 \times 10^{-4}$ \\
$h_{1}(1380)$ & $3.8 \times 10^{-5}$ \\
$K_{1}(1)$ & $2.1 \times 10^{-5}$ \\
$K_{1}(2)$ & $4.1 \times 10^{-6}$ \\
\hline \hline
\end{tabular}

calculation, since the sources of uncertainty are similar to those of Ref. [13], we can also expect an error of the order of $40 \%$ to the values shown in Table VI. Of the branching ratios calculated in the present work only the one for $\tau^{-} \rightarrow \nu_{\tau} \pi^{-} f_{1}(1285)$ has been experimentally measured [5] giving $(3.8 \pm 1.4) \times 10^{-4}$, which compares well with the value we obtain for that channel within uncertainties. For the channels not yet measured, even though the branching ratios obtained for some of them seem small, they are of the same order as many of the already experimentally measured hadronic decays reported by the PDG [5]. We can also compare our results with previous theoretical works which also tackle these decays. For instance, in Ref. [22] the decay of the $\tau$ into an axial and a pseudoscalar was evaluated considering a intermediate production of a $\rho(770)$ and a $\rho(1450)$ from the $W$ boson and then decaying into the final axial and pseudoscalar. However all the couplings involved were obtained experimentally. Concerning the different channels, the results obtained here agree for $a_{1} \pi, h_{1}(1170) \pi$ and $f_{1}(1285) \pi$ with those of [22] within uncertainties, while for the $K_{1}(1270)$ channels they disagree by 3-4 orders of magnitude. Note, however, that the discrepancy in the $K_{1}(1270)$ results is expectable since the model we use for the axial vector mesons predicts that there are actually two poles for the $K_{1}(1270)$. In Refs. [23,24] only the $f_{1}(1285) \pi$ channel was evaluated using a Nambu-Jona-Lasinio model and they found a branching ratio of $3.98 \times 10^{-4}$, compatible within errors with our result.

The mass distributions and the branching ratios of Table VI are nontrivial and genuine predictions because, first, they crucially depend on the axial-vector resonance couplings to VP which are a nontrivial output of the chiral unitary model [11] and consequence of the dynamical origin of these resonances, and, second, because of the nontrivial shape of the triangular mechanism and the enhancement due to nearby singularities when present. Therefore, experimental measurements of these decays could check the dynamical origin of these axial-vector resonances.

\section{TRIANGLE MECHANISM WITH TWO INTERMEDIATE PIONS}

For the states of negative $G$-parity $h_{1}(1170), h_{1}(1380)$ and $a_{1}(1260)$ we can consider the diagrams of Fig. 1 but replacing the $\rho$ by pions and the pions by $\rho$, as shown in Fig. 15. The calculation is done following the same steps as before, with several changes. The first one is that the $\tau^{-} \rightarrow \nu_{\tau} \pi^{-} \pi^{0}$ proceeds via $p$-wave. According to Ref. [19] the $M_{0}$ term is zero and $N_{i}$ is given by

$$
N_{i}=\bar{h} \frac{1}{\sqrt{2 \pi}} \tilde{p}_{1 i} ; \quad \bar{h}=\sqrt{2}
$$

and $\tilde{p_{1}}$ is the $\pi^{-}$momentum in the $\pi^{-} \pi^{0}$ rest frame. In this case the differential decay width is given by

$$
\frac{d \Gamma}{d M_{\text {inv }}(\pi \pi)}=\frac{2 m_{\tau} 2 m_{\nu}}{(2 \pi)^{3}} \frac{1}{4 m_{\tau}^{2}} p_{\nu} \tilde{p}_{1} \sum \sum|t|^{2},
$$

with

$$
\begin{aligned}
& p_{\nu}=\frac{\lambda^{1 / 2}\left(m_{\tau}^{2}, m_{\nu}^{2}, M_{\mathrm{inv}}^{2}(\pi \pi)\right)}{2 m_{\tau}}, \\
& \tilde{p}_{1}=\frac{\lambda^{1 / 2}\left(M_{\mathrm{inv}}^{2}(\pi \pi), m_{\pi}^{2}, m_{\pi}^{2}\right)}{2 M_{\mathrm{inv}}(\pi \pi)},
\end{aligned}
$$

and

$$
\bar{\sum} \sum|t|^{2}=\mathcal{C}^{\prime 2} \bar{h}^{2} \frac{1}{m_{\tau} m_{\nu}} \frac{1}{2 \pi}{\tilde{p_{1}}}^{2}\left(E_{\tau} E_{\nu}-\frac{p^{2}}{3}\right)
$$

and $p$ is the momentum of $\tau$ or $\nu_{\tau}$ in the $\pi \pi$ rest frame

$$
p=\frac{\lambda^{1 / 2}\left(m_{\tau}^{2}, m_{\nu}^{2}, M_{\mathrm{inv}}^{2}(\pi \pi)\right)}{2 M_{\mathrm{inv}}(\pi \pi)},
$$

with $E_{\tau}, E_{\nu}$ evaluated in that frame.

By integrating $\frac{d \Gamma}{d M_{\text {inv }}(\pi \pi)}$ we can get the $\tau^{-} \rightarrow \nu_{\tau} \pi^{+} \pi^{-}$ width, and comparing it with the experimental branching ratio from the non- $\rho \rightarrow \pi^{-} \pi^{0}, \quad \mathcal{B}\left(\tau \rightarrow \nu_{\tau} \pi^{-} \pi^{0}\right)=$ $(3.0 \pm 3.2) \times 10^{-3}$, we can determine the normalization 


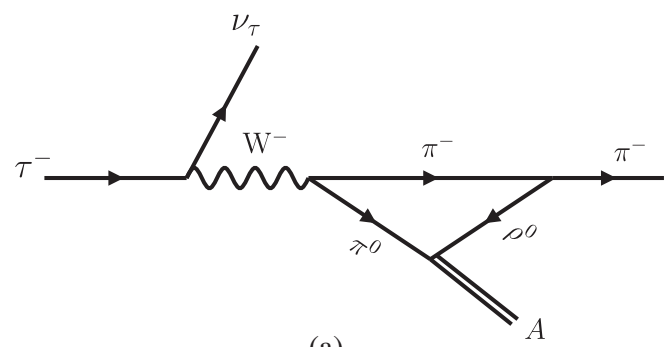

(a)

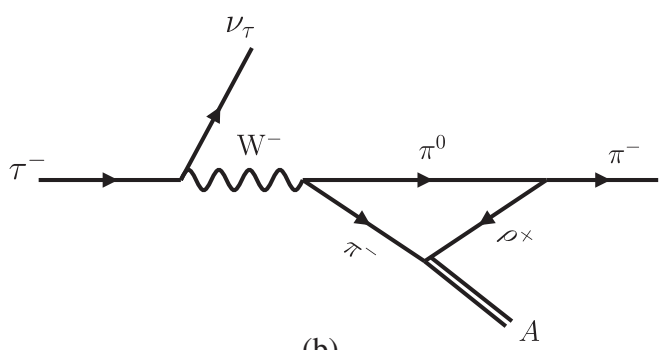

(b)

FIG. 15. Diagrams for the decay of $\tau^{-} \rightarrow \nu_{\tau} \pi^{-} A$ exchanging pions and rhos. The momenta assignment is the same as Fig. 2 for the decay process.

constant $\mathcal{C}^{\prime 2}$ Certainly, with this large experimental error we can only make an estimate of the final contribution of our process.

In order to evaluate the contribution of the diagrams of Fig. 15, we must reevaluate the loop function because in $t_{L}$ of Eq. (27) only the positive energy part of the vector propagator is taken. Here we do the same but the vector is now in the position of particle 3, with different kinematics than in position 1 in the former diagrams. One must also consider that in the diagram (a) of Fig. 15 the $\pi^{-}$has momentum $-\boldsymbol{q}$, while in the diagram (b) it has momentum $\boldsymbol{q}$. This sign is compensated by a different sign in the $\pi^{-} \rightarrow \pi^{-} \rho^{0}, \pi^{0} \rightarrow \pi^{-} \rho^{+}$vertices, such that we get the same combinations of couplings as in Eq. (39).
At the end we find that

$$
N_{i}=F\left\{k_{i} \boldsymbol{\epsilon}_{\boldsymbol{A}} \cdot \boldsymbol{k} t_{L}^{(1)}+\epsilon_{A_{i}} t_{L}^{(2)}\right\},
$$

where $F$ collects all the factors,

$$
F=\frac{\bar{h}}{\sqrt{2 \pi}} \sqrt{2} g B g_{A, \rho \pi} ; \quad B=\left\{\begin{array}{ll}
\frac{2}{\sqrt{3}}, & \text { for } I=0\left(h_{1}\right) \\
-\frac{1}{\sqrt{2}}, & \text { for } I=1\left(a_{1}\right)
\end{array},\right.
$$

and

$$
\begin{aligned}
t_{L}^{(1)}= & \int \frac{d^{3} q}{(2 \pi)^{3}}\left\{\frac{\boldsymbol{k} \cdot \boldsymbol{q}}{\boldsymbol{k}^{2}}-\frac{3}{2} \frac{(\boldsymbol{k} \cdot \boldsymbol{q})^{2}}{\boldsymbol{k}^{4}}+\frac{1}{2} \frac{\boldsymbol{q}^{2}}{\boldsymbol{k}^{2}}\right\} \frac{1}{8 \omega_{1} \omega_{2} \omega} \\
& \times \frac{2\left(\omega_{1}+\omega_{2}\right)\left(\omega_{1}+\omega_{2}+\omega+k^{0}\right)-2 P^{0} \omega_{2}}{\left(\omega_{1}+k^{0}+\omega\right)\left(P^{0}+\omega_{1}+\omega_{2}\right)\left(P^{0}-\omega_{1}-\omega_{2}+i \epsilon\right)\left(P^{0}-k^{0}-\omega-\omega_{2}+i \epsilon\right)}, \\
t_{L}^{(2)}= & \int \frac{d^{3} q}{(2 \pi)^{3}} \frac{1}{2}\left\{\frac{(\boldsymbol{k} \cdot \boldsymbol{q})^{2}}{\boldsymbol{k}^{2}}-\boldsymbol{q}^{2}\right\} \frac{1}{8 \omega_{1} \omega_{2} \omega} \\
& \times \frac{2\left(\omega_{1}+\omega_{2}\right)\left(\omega_{1}+\omega_{2}+\omega+k^{0}\right)-2 P^{0} \omega_{2}}{\left(\omega_{1}+k^{0}+\omega\right)\left(P^{0}+\omega_{1}+\omega_{2}\right)\left(P^{0}-\omega_{1}-\omega_{2}+i \epsilon\right)\left(P^{0}-k^{0}-\omega-\omega_{2}+i \epsilon\right)},
\end{aligned}
$$

where

$$
\omega=\sqrt{(\boldsymbol{q}+\boldsymbol{k})^{2}+m_{\rho}^{2}}, \quad \omega_{1}=\sqrt{m_{\pi}^{2}+\boldsymbol{q}^{2}}, \quad \omega_{2}=\sqrt{m_{\pi}^{2}+\boldsymbol{q}^{2}},
$$

and $i \epsilon$ is replaced by $i \frac{\Gamma_{\rho}}{2}$ in the last denominator of Eq. (59).

\footnotetext{
${ }^{2}$ The part of the $\tau^{-} \rightarrow \nu_{\tau} \rho^{-}\left(\rho^{-} \rightarrow \pi^{0} \pi^{-}\right)$decay is not considered because in the diagrams of Fig. 15 it would introduce the $\rho$ propagator with a $\rho$ invariant mass equal to $M_{\text {inv }}\left(\pi^{-} A\right)$, of the order of $1400 \mathrm{MeV}$, for which the $\rho$ propagator $\left(M_{\mathrm{inv}}^{2}\left(\pi^{-} A\right)-\right.$ $\left.m_{\rho}^{2}+i m_{\rho} \Gamma_{\rho}\right)^{-1}$ is highly suppressed.
}

Contracting $N_{i} N_{j}^{*}$ with $L^{i} L^{j \dagger}$ of Eq. (7) we find

$$
\begin{aligned}
\overline{\sum \sum|t|^{2}=} & \frac{F^{2}}{m_{\tau} m_{\nu}}\left(E_{\tau} E_{\nu}-\frac{1}{3} p^{2}\right)\left\{3\left|t_{L}^{(2)}\right|^{2}+k^{2}\left|t_{L}^{(1)}\right|^{2}\right. \\
& \left.+t_{L}^{(1)} t_{L}^{(2) *}+t_{L}^{(1) *} t_{L}^{(2)}\right\}
\end{aligned}
$$

and now

$$
\frac{1}{\Gamma_{\tau}} \frac{d \Gamma}{d M_{\mathrm{inv}}\left(\pi^{-} A\right)}=\frac{\mathcal{C}^{\prime 2}}{\Gamma_{\tau}} \frac{1}{(2 \pi)^{3}} \frac{2 m_{\tau} 2 m_{\nu}}{4 m_{\tau}^{2}} p_{\nu} \tilde{p}_{\pi} \bar{\sum} \sum|t|^{2},
$$




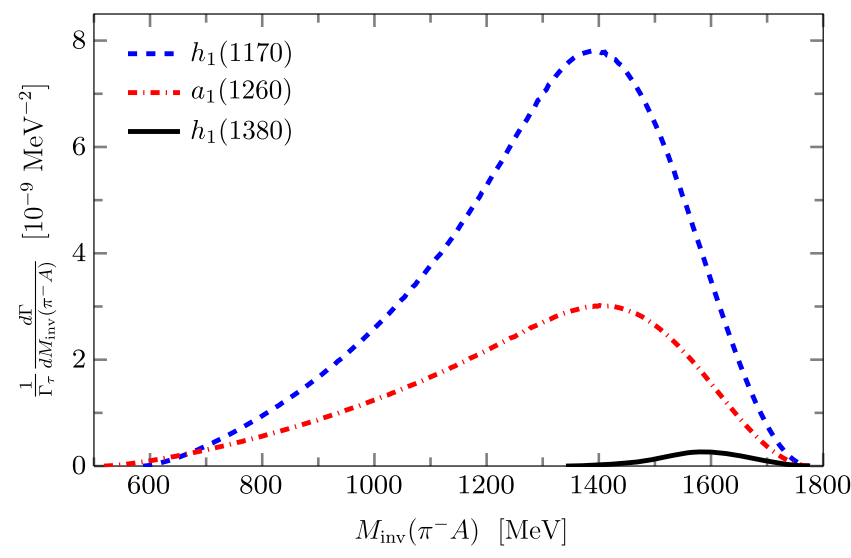

FIG. 16. The mass distribution of $\tau^{-} \rightarrow \nu_{\tau} \pi^{-} A$ decay from the mechanism of Fig. 15.

with

$$
\begin{aligned}
& p_{\nu}=\frac{\lambda^{1 / 2}\left(m_{\tau}^{2}, m_{\nu}^{2}, M_{\mathrm{inv}}^{2}\left(\pi^{-} A\right)\right)}{2 m_{\tau}}, \\
& \tilde{p}_{\pi}=k=\frac{\lambda^{1 / 2}\left(M_{\mathrm{inv}}^{2}\left(\pi^{-} A\right), m_{\pi}^{2}, m_{A}^{2}\right)}{2 M_{\mathrm{inv}}\left(\pi^{-} A\right)},
\end{aligned}
$$

and $p$ the $\tau, \nu_{\tau}$ momentum in the $\pi^{-} A$ rest frame given by Eq. (48), with $E_{\tau}, E_{\nu}$ evaluated in the same frame. To the mass distribution of Eq. (62) we have also implemented its folding with the axial-vector spectral function as explained in the previous section.

In Fig. 16 we show the invariant mass distributions of the different $\tau^{-} \rightarrow \nu_{\tau} \pi^{-} A$ decays by exchanging two intermediate pions.

In Table VII we show the integrated branching ratios of exchanging two intermediate pions for the different $\tau \rightarrow$ $\nu_{\tau} \pi^{-} A$ decay channels.

The results are obtained using the central value of the non- $\rho \mathcal{B}\left(\tau \rightarrow \nu_{\tau} \pi^{-} \pi^{0}\right)=3.0 \times 10^{-3}$ and they are about three orders of magnitude smaller than the results obtained from the previous mechanisms. Even if we have to sum the amplitudes and not the widths, the structures are rather different indicating small interference, but even with maximum interference, one can only get a few percent contribution from this mechanism.

TABLE VII. The branching ratios of exchanging two intermediate pions.

\begin{tabular}{cc}
\hline \hline & $\mathcal{B}$ \\
\hline$h_{1}(1170)$ & $4.0 \times 10^{-6}$ \\
$a_{1}(1260)$ & $1.7 \times 10^{-6}$ \\
$h_{1}(1380)$ & $5.1 \times 10^{-8}$ \\
\hline \hline
\end{tabular}

\section{CONCLUSIONS}

We have carried out a theoretical study of the $\tau$ decay into a pseudoscalar meson plus an axial-vector resonance. These hadronic decay channels have been very little studied previously, both theoretical and experimentally. Nonetheless, these channels could play an important role in order to shed light on the dynamical formation and structure of the axial-vector resonances. In particular we focused on the two lowest mass nonets of axial-vector mesons, $a_{1}(1260) b_{1}(1235), \quad h_{1}(1170), \quad h_{1}(1380), \quad a_{1}(1260)$, $f_{1}(1285)$, and both poles of the $K_{1}(1270)$. There has been in the last 15 years compelling theoretical and experimental evidence that these resonances can be interpreted as molecular or dynamically generated from the interaction of a pseudoscalar and a vector meson in s-wave. Indeed, using the techniques of the chiral unitary approach $(U \chi P T)$, which extends the range of applicability of $\chi P T$ beyond the lowest resonance regions by the implementation of unitarity in coupled channels to a lowest order amplitude obtained from chiral Lagrangians, poles of the unitarized PV amplitudes were found which could be associated to the known axialvector resonances. In particular, of great relevance was the prediction that in the strange sector the $K_{1}(1270)$ actually corresponds to two disctinct poles with different coupling intensities to the different VP channels. Within this framework the dominant production mechanism for the $\tau$ decays considered in the present work is through a triangle mechanism of the kind shown in Fig. 1, since a vector and a pseudoscalar need to be produced, in addition to the extra final pseudoscalar, to generate the axial-vector resonance. The initial VV production from the weak current has been theoretically determined, up to a global common factor obtained from the experimental $\tau \rightarrow \nu_{\tau} K^{* 0} K^{*-}$ branching ratio, from a primary $d \bar{u}$ formation from the $W^{-}$boson which then hadronizes producing an extra $q \bar{q}$ pair within the ${ }^{3} P_{0}$ model. The spinor algebra is worked out following a recent approach where different $G$-parity contributions could be easily filtered, of special interest in the present work.

The pseudoscalar-axial mass distributions predicted in the present work manifest shifts of the strength to the higher energy region of the spectrum partly due to the special shape of the triangle loop function which is carefully evaluated. We make predictions also for integrated branching ratios and, for the only channel experimentally measured, $\tau^{-} \rightarrow \nu_{\tau} \pi^{-} f_{1}(1285)$, our result agree with it within uncertainties. For most of the other channels, the strength of the predicted branching ratios are such that they should be expected to be attainable in experimental studies devoted to exclusive hadronic $\tau$ decays. Since the strength of the decays depends crucially on the coupling of the axialvector resonances to the different VP channels, and these are genuine and nontrivial predictions of the $U \chi P T$ approach, a positive comparison with those experimental results should reinforce the dynamical or molecular nature of these axial-vector resonances. 


\section{ACKNOWLEDGMENTS}

L. R. D. acknowledges the support from the National Natural Science Foundation of China (Grant No. 11575076) and the State Scholarship Fund of China (No. 201708210057). This work is partly supported by the
Spanish Ministerio de Economia y Competitividad and European FEDER funds under Contracts No. FIS201784038-C2-1-P B and No. FIS2017-84038-C2-2-P B, and the Generalitat Valenciana in the program Prometeo II-2014/068, and the project Severo Ochoa of IFIC, SEV-2014-0398 (EO).
[1] S. Schael et al. (ALEPH Collaboration), Phys. Rep. 421, 191 (2005).

[2] M. Davier, A. Hocker, and Z. Zhang, Rev. Mod. Phys. 78, 1043 (2006).

[3] E. Braaten, S. Narison, and A. Pich, Nucl. Phys. B373, 581 (1992).

[4] G. D. Lafferty, Nucl. Part. Phys. Proc. 260, 247 (2015).

[5] M. Tanabashi et al. (Particle Data Group), Phys. Rev. D 98, 030001 (2018).

[6] D. Boito, M. Golterman, K. Maltman, J. Osborne, and S. Peris, Phys. Rev. D 91, 034003 (2015).

[7] A. Pich, Prog. Part. Nucl. Phys. 75, 41 (2014).

[8] J. Portoles, Nucl. Phys. B, Proc. Suppl. 169, 3 (2007).

[9] M. Davier, A. Hcker, B. Malaescu, C. Z. Yuan, and Z. Zhang, Eur. Phys. J. C 74, 2803 (2014).

[10] M. F. M. Lutz and E. E. Kolomeitsev, Nucl. Phys. A730, 392 (2004).

[11] L. Roca, E. Oset, and J. Singh, Phys. Rev. D 72, 014002 (2005).

[12] Y. Zhou, X. L. Ren, H. X. Chen, and L. S. Geng, Phys. Rev. D 90, 014020 (2014).

[13] E. Oset and L. Roca, Phys. Lett. B 782, 332 (2018).

[14] L. D. Landau, Nucl. Phys. 13, 181 (1959).

[15] S. Coleman and R. E. Norton, Nuovo Cimento 38, 438 (1965).

[16] M. Bayar, F. Aceti, F. K. Guo, and E. Oset, Phys. Rev. D 94, 074039 (2016).
[17] V. R. Debastiani, S. Sakai, and E. Oset, Eur. Phys. J. C 79, 69 (2019).

[18] L. R. Dai, Q. X. Yu, and E. Oset, Phys. Rev. D 99, 016021 (2019).

[19] L. R. Dai, R. Pavao, S. Sakai, and E. Oset, Eur. Phys. J. A 55, 20 (2019).

[20] A. Le Yaouanc, L. Oliver, O. Pene, and J. C. Raynal, Phys. Rev. D 8, 2223 (1973).

[21] F. E. Close, An Introduction to Quark and Partons (Academic Press, New York, 1979).

[22] G. Calderon, J. H. Munoz, and C. E. Vera, Phys. Rev. D 87, 114011 (2013).

[23] A. V. Vishneva, M. K. Volkov, and D. G. Kostunin, Eur. Phys. J. A 50, 137 (2014).

[24] M. K. Volkov, A. A. Pivovarov, and A. A. Osipov, Eur. Phys. J. A 54, 61 (2018).

[25] L. S. Geng, E. Oset, L. Roca, and J. A. Oller, Phys. Rev. D 75, 014017 (2007).

[26] L. Micu, Nucl. Phys. B10, 521 (1969).

[27] E. Santopinto and R. Bijker, Phys. Rev. C 82, 062202 (2010).

[28] F. Mandl and G. Shaw, Quantum Field Theory (John Wiley \& Sons, New York, 1984).

[29] S. Sakai, E. Oset, and A. Ramos, Eur. Phys. J. A 54, 10 (2018).

[30] F. Aceti, J. M. Dias, and E. Oset, Eur. Phys. J. A 51, 48 (2015). 